AperTO - Archivio Istituzionale Open Access dell'Università di Torino

\title{
Inflammatory and redox reactions in colorectal carcinogenesis
}

\section{This is a pre print version of the following article:}

Original Citation:

\section{Availability:}

This version is available http://hdl.handle.net/2318/1509271

since 2016-07-20T18:18:22Z

Published version:

DOI:10.1111/nyas.12734

Terms of use:

Open Access

Anyone can freely access the full text of works made available as "Open Access". Works made available under a Creative Commons license can be used according to the terms and conditions of said license. Use of all other works requires consent of the right holder (author or publisher) if not exempted from copyright protection by the applicable law. 


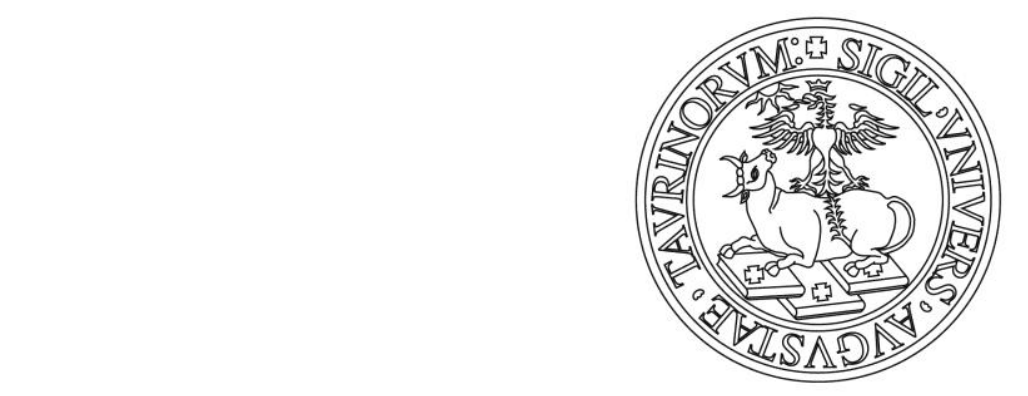

\section{UNIVERSITÀ DEGLI STUDI DI TORINO}

This is the pre-print version of the following article: [Inflammatory and redox reactions in colorectal carcinogenesis], which has been published in final form at [http://onlinelibrary.wiley.com/doi/10.1111/nyas.12734/abstract;jsessionid=B6E63 06E4BFA6A476D1AFD7242CB52C3.f01t02], Annals of the New York Academy of Sciences, Volume 1340, Cellular and Environmental Stressors in Biology and Medicine pages 95-103, March 2015. 
Short title: Inflammation and oxidative stress in CRC

Full title: Inflammatory and redox reactions in colorectal carcinogenesis

Tina Guina ${ }^{1}$, Fiorella Biasi ${ }^{1}$, Simone Calfapietra ${ }^{1}$, Mario Nano ${ }^{2}$, Giuseppe Poli ${ }^{1}$

${ }^{1}$ Department of Clinical and Biological Sciences at San Luigi Hospital, University of Turin, Italy

${ }^{2}$ Department of Oncology at San Luigi Hospital, University of Turin, Italy

\section{Corresponding author:}

Fiorella Biasi

Department of Clinical and Biological Sciences, University of Turin at San Luigi Gonzaga

Hospital, 10043 Orbassano (Turin), Italy

Phone number: 00390116705420 ; Fax number: 00390116705424

E-mail: fiorella.biasi@unito.it

Key words: colorectal cancer; inflammatory bowel disease; reactive oxygen species; antioxidant response 


\section{$\underline{\text { Abstract }}$}

It is established that there is a relationship between inflammation and cancer development. The constant colonic inflammation typical of inflammatory bowel diseases is now considered a risk factor for colorectal cancer (CRC) development. The inflammatory network of signaling molecules is also required during the late phases of carcinogenesis, to enable cancer cells to survive and to metastasize. Oxidative reactions are an integral part of the inflammatory response, and are generally associated to CRC development. However, when the malignant phenotype is acquired, increased oxidative status induces antioxidant defenses in cancer cells, favoring their aggressiveness. This contradictory behavior of cancer cells towards redox status is of great significance for potential anticancer therapies.

The paper summarizes the essential background information relating to the molecules involved in regulating oxidative stress and inflammation during carcinogenesis. Today's understanding of their function in CRC stages might provide the foundations for future developments in CRC treatment. 


\section{$\underline{\text { Introduction }}$}

Colorectal carcinoma (CRC) is the third commonest cancer, with almost 1.4 million new cases and 0.7 million deaths worldwide in 2012; 500,000 new cases and 250,000 deaths have been reported in Europe. In Western countries, for incidence and mortality CRC is the second malignant neoplasm after breast cancer in women, and the third after lung and prostate cancers in men. Onset is rare before the age of 40; it is more frequent in older people, with a peak around the age of 80 . The incidence of CRC is now increasing in the female population, because of lifestyle, which has become more uniform between the two sexes. ${ }^{1}$

Inflammation has been considered to play a major role in the initiation, promotion and progression of CRC: the constant colonic inflammation typical of inflammatory bowel disease (IBD) may lead to Colitis-Associated Cancer (CAC), a subtype of CRC. More than $20 \%$ of patients with IBD develop CAC within 30 years of the disease onset, and more than $50 \%$ of them die as a result of malignant transformation. ${ }^{2} \mathrm{CAC}$ usually affects younger persons, and is characterized by multiple lesions, located in more proximal segments of the colon mucosa compared to sporadic CRC. The duration of active IBD and the extent of inflamed colonic mucosa have been identified as two major risk factors for developing CRC. Further, the risk of developing cancer is much higher in IBD patients with primary sclerosing cholangitis, which is a predisposing factor for cancer of the biliary tract. The administration of non-steroidal anti-inflammatory drugs, especially 5-aminosalicylates, can reduce the development of dysplasia and colorectal carcinoma. ${ }^{3}$

Infiltration and enhanced activation of inflammatory cells may initiate and further promote cancer development. These cells generate inflammatory cytokines, several of which play crucial roles in CRC carcinogenesis. Further, chronic inflammation in IBD exposes the intestinal mucosa to severe oxidative insult, which increases the risk of malignant transformation, promoting cell mutation and cancer proliferation. An inappropriate response of the mucosa-associated immune system to commensal and pathogenic microbiota, as occurs in IBD, may contribute to inducing excessive inflammatory and oxidative reactions by activated leukocytes, thus amplifying intestinal mucosa 
injury. Neutrophils and macrophages generate free radicals and other pro-oxidant molecules: the inflamed tissue of patients with active IBD shows an increased expression of reactive oxygen species (ROS). Oxidative stress and the associated mucosal damage play key roles in the pathogenesis of colitis, as well as in colorectal carcinogenesis. ${ }^{4,5}$

This review will focus on the roles of inflammation and oxidative stress in CRC initiation and progression. Understanding how the molecules involved in these processes can function as sensors and modulators of cancer behavior may contribute to providing new therapeutic strategies against the development and progression of CRC.

\section{$\underline{\text { Inflammatory cells and CRC }}$}

CRC may be taken as a model of the link between inflammation and cancer. Inflammation is driven by cytokines and chemokines, which may be produced by the tumor cells themselves or, more often, by cells recruited to the tumor microenvironment.

Lymphocytic infiltration in CRC was thought to be associated with a better prognosis. ${ }^{6}$ The immunosurveillance system may recognize and eliminate the transformed malignant cells, or even small tumors in a quiescent state; this process may also lead to the deletion of single cells or small groups of metastatic cells. Conversely, lymphocytic infiltrate has been shown to promote carcinogenesis. It has been shown that adaptive immune response cells, recruited in CRC, can display either pro- or anti-tumoral activity. $\mathrm{T}$ lymphocytes may favor the development and progression of tumor-activating inflammation, whereas conversely they may act against the tumor, through immunosurveillance. $^{7}$

Similar activity has been observed in Tumor Associated Macrophages (TAM), whose polarization from the pro-inflammatory M1 to the pro-tumoral M2 phenotype has been confirmed to be a key event in the carcinogenesis of many malignant tumors, including CRC. The M2 phenotype produces several molecules that sustain malignant cell survival and proliferation, suppress the anti-tumoral adaptive immune response, modify extracellular matrix (ECM) proteins, and promote 
neoangiogenesis. Interestingly, the concomitant presence of both TAM phenotypes has been observed at the tumor front: the M1/ M2 macrophage ratio might be an important prognostic index for CRC patients. ${ }^{8}$

There is some evidence that neutrophils may be associated with carcinogenesis, in the form of Tumor Associated Neutrophils (TAN), by acquiring the N2 pro-tumoral phenotype, similarly to macrophages. TAN tumor infiltration may be responsible for genetic instability, through the production of ROS, and may facilitate invasion, through the release of a large number of serine proteases and chemokines. ${ }^{9}$ The important role played by TAN in tumor initiation and progression has been demonstrated in experimental models of colitis associated to carcinogenesis in mice ${ }^{10}$ and in humans. ${ }^{11}$

\section{The key role of inflammatory molecules}

A large number of inflammatory molecules are involved during different steps of carcinogenesis; they act by influencing tumor cell growth, differentiation, and survival.

The molecules most widely known to be involved in the inflammatory process are the ecosanoids (prostaglandins, prostacyclins, thromboxanes, and leukotrienes). In particular, increased activity of the inducible enzyme cyclooxygenase-2 (COX-2), which catalyzes prostaglandin (PG) synthesis, plays an important role in CRC carcinogenesis. Increased COX-2 expression has been detected in CAC animal models, ${ }^{12}$ as well as in the tissue of CRC patients, where it is associated to poor prognosis. ${ }^{13}$ The pro-neoplastic effect of COX-2 appears to be mediated by its major end product, PGE2, which can stimulate the tumor microenvironment to induce cancer stem cells. ${ }^{14}$

Besides the classical inflammatory mediators just mentioned, cytokines and chemokines may act as tumor growth factors, and may, together with the serine proteinases, promote tumor invasion by inducing angiogenesis and suppressing the antitumor immune response. Specific pro-inflammatory cytokines, such as interleukins (ILs) IL-1, IL-6, IL-8 and Tumor Necrosis Factor- $\alpha$ (TNF- $\alpha$ ), or the transcription factors required for the signals induced by these cytokines, including Nuclear Factor- 
$\kappa \mathrm{B}(\mathrm{NF}-\kappa \mathrm{B})$ and Signal Transducer and Activator of Transcription (STAT) 3, are thus now emerging as potential targets for anticancer therapy. ${ }^{12}$

Although the first studies that found evidence for tumor promotion regulated by cytokines were on CAC, the same mechanisms may also apply to sporadic CRC: cytokines IL-1 $\beta$, IL-6, and TNF- $\alpha$ have been shown to promote the development of both CAC and sporadic CRC. ${ }^{15}$

TNF- $\alpha$, which is produced during the early inflammatory response, increases vascular permeability, leading to recruitment of activated leukocytes at the tumor site. ${ }^{12}$ Notably, several different commensal and pathogenic bacterial strains have been found to activate TNF- $\alpha$ and IL- 8 in IBD patients. ${ }^{16}$

IL-8, with its receptor CXCR2, is the most strongly up-regulated cytokine in CRC. It enhances the proliferation and survival of cancer cells through autocrine activation, and promotes angiogenesis and neutrophil infiltration into the tumor. IL-8 is abundantly synthesized by N2 neutrophils, ${ }^{9}$ and its expression has been found to be elevated in patients with metastatic disease. ${ }^{17}$ It plays an important role in the process of tumor invasion, because of its direct involvement in up-regulating the expression of ECM serine proteinases, such as Matrix Metalloproteinases (MMPs). A positive correlation between IL-8 and MMP-9 has been reported in advanced stages of CRC. ${ }^{18}$

IL-6 plays an important role in inflammation related to CRC pathogenesis: high levels of this cytokine have been detected in IBD, as well as in CRC patients. Most of the effects of IL-6 on cancer cells may be due to interleukin's ability to activate the JAK/STAT signaling pathway. ${ }^{19}$ Specific cytokines produced by tumor infiltrating T helper (Th) 17 CD4+ lymphocytes, such as IL17 and IL22, have been reported to be increased in experimental and human CRC; together with IL6 and TNF- $\alpha$, these cytokines activate STAT3 and NF- $\mathrm{KB}$, and contribute to tumor promotion and progression. Inhibition of these transcription factors, in particular of STAT3, might down-regulate the production of cytokines by tumor infiltrating cells, consequently inhibiting CRC growth. ${ }^{20,15}$ IL-23 is a cytokine that promotes Th17 cell development and survival and IL-17 related synthesis. Specific polymorphisms of the gene encoding for IL-23 receptor, as well as that for IL-17, have 
been associated with Crohn's disease (CD), ulcerative colitis UC), ${ }^{4}$ and some clinical features of human CRC. ${ }^{21}$

Transforming Growth Factor $\beta$ (TGF $\beta$ ) is a cytokine whose role in tumor development is paradoxical: it exhibits antitumor effects in the earliest phases of tumor growth, by inhibiting proliferation, promoting apoptosis, and suppressing the expression of tumor promoting proinflammatory cytokines. It is widely accepted that mutations of the TGF $\beta$ pathway in epithelial cells predispose to, or facilitate, CRC development and growth. ${ }^{22}$ Conversely, during the later phases of tumor progression, the tumor cells require the TFG $\beta$ function in order to promote EpithelialMesenchymal Transition and suppress the immune cells' antitumor activity, thus facilitating metastasis. ${ }^{23}$ Notably, TANs acquire the N2 pro-tumoral phenotype in response to TGF $\beta .{ }^{9}$

MMPs, in particular the gelatinases MMP-9 and MMP-2, are serine proteinases whose main function is to degrade collagen IV, a major component of the basement membranes of epithelial tissues. MMP activity is essential in CRC development and invasiveness. During carcinogenesis, the main sources of production of MMPs are M2 macrophages and stromal cells, ${ }^{8}$ particularly in advanced stages of CRC.

Our own experience with CRC patients has shown that MMP-9 levels are significantly higher in CRCs at stages III and IV (TNM classification), in both the peripheral blood and tumor tissue. A direct relation was also present among MMP-9, IL-8, and C reactive protein serum levels, all of which increased at more advanced tumor stages. Based on these findings, these molecules might be considered as reliable indexes of inflammation during CRC progression. ${ }^{18}$ Further, as-yet unpublished data confirm MMP-9 to be a reliable diagnostic factor for CRC, since preoperative MMP-9 serum levels effectively discriminated healthy subjects from cancer patients. However, MMP-9 alone showed a poor capacity to predict the onset of metastasis, independently of disease stage, at different times after surgical removal of the tumor mass. 
Inflammation might be the link between oxidative stress and $\mathrm{CRC}$, in which ROS are overproduced by activated leukocytes. ROS ant their oxidation products could thereby overwhelm the tissue's antioxidant defenses, and contribute to the functional impairment that in all likelihood leads to dysplasia of the enteric mucosa. Inflammatory phagocytic cells, such as neutrophils and macrophages, present on their surface the enzyme responsible for the "respiratory burst", namely NADPH oxidase (NOX); this enzyme produces the ROS required for processing and killing bacteria. Upon contact with pathogens or specific cytokines, NOX converts oxygen to superoxide $\left(\mathrm{O} 2 \bullet^{-}\right)$which is then converted by antioxidant enzymes to $\mathrm{H} 2 \mathrm{O} 2$ through superoxide dismutase (SOD), and to $\mathrm{H} 2 \mathrm{O}$ and $\mathrm{O} 2$ through glutathione peroxidase (GPX) and catalase. Intestinal NOX1 isoform, as well as NOX complex members NOX Organizer 1 (NOXO1) and NOX Activator 1 (NOXA1), are reported to be significantly overexpressed in CRC tissue compared to adjacent normal colon mucosa. ${ }^{24}$

The phagocytic isoform, NOX2, is potently activated by $\mathrm{TNF} \alpha$, and NoxOl gene transactivation induced by this cytokine is reported in human colon epithelial cells, suggesting that this oxidase may be a potential target in colon inflammation. ${ }^{25}$

Reactive nitrogen species (RNS) that are produced by phagocytes during inflammation, i.e. nitric oxide (NO), through inducible nitric oxide synthase (iNOS) and peroxynitrite (OONO), have also been implicated in cancer development. The oxidation of various cellular macromolecules and the modulation of gene expression, both brought about by these reactive species, are thought to be one of the main mechanisms of cancer initiation. ${ }^{5}$

The induction of DNA mutations by ROS attack is considered to be principally involved in the early events of colon carcinogenesis linked to inflammatory processes. Reaction of the hydroxyl radical at the C-8 position of deoxyguanosine induces formation of 8-hydroxy-2'-deoxyguanosine (8$\mathrm{OHdG}$ ), which might play a role in mutagenic events and genetic instability in the early phases of cancer initiation and promotion. Levels of this marker of oxidative DNA damage have been found to be twice normal in experimental colitis induced in rats treated with dextran sodium sulfate (DSS). 
Concomitant administration of the COX-2 inhibitor nimesulide clearly demonstrated a net decrease in $8-\mathrm{OHdG}$ production in colonocytes. ${ }^{26}$ Increased levels of $8-\mathrm{OHdG}$ have been found in the plasma of patients suffering from $\mathrm{UC}$ or $\mathrm{CD} .{ }^{27} \mathrm{NO}$-dependent oxidative DNA lesions producing G:C to $\mathrm{T}$ :A base transversions have been associated to TP53 mutations in inflamed colon portion of UC patients. $^{28}$

Mitochondria are the main sites for ROS generation together with NADPH oxidase. Mitochondrial DNA (mtDNA) is thus particularly exposed to ROS damage, being localized close to the intracellular mitochondria electron transport chain. Increased mutations of mtDNA, and the high 8OHdG tissue concentrations found in mucosal specimens from patients with UC, have been related to the high incidence of CRC in these subjects. ${ }^{29}$

Protein oxidation leads to the alteration of both structural and functional proteins, which are involved in the maintenance of cell integrity. Special attention should be paid to the oxidative modification of enzymes that may be inhibited in specific activities, including inactivation of the DNA mismatch repair system, thus damaging the protein complex responsible for DNA repair. ${ }^{5}$

Polyunsaturated fatty acids (PUFAs) are highly susceptible to free radical attack on double bonds, whose breaking and rearrangement leads to the generation of more stable aldehyde end-products, able to react with cellular structures. In particular, the aldehydes produced during the oxidative breakdown of PUFA have been considered as final mediators of the toxic effects elicited by lipid peroxidation in cells and tissues. Arachidonic acid is the $\omega 6$-PUFA most closely involved in both inflammation (being the substrate for the enzymatic production of prostaglandins through COXs activity) and lipid peroxidation: its breakdown generates the end-product 4-hydroxy-2,3-nonenal (HNE), which has been shown to react more strongly than do other aldehydes with biomolecules containing amino and thiol groups, thus modifying the proteins involved in cell signaling pathways that induce the antioxidant response. Rapid conjugation of HNE with glutathione (GSH) markedly depletes this antioxidant in different cell types. Further, HNE reacts with all four DNA bases, in particular making more efficient adducts with deoxyguanosine. ${ }^{30}$ 
Oxidative-stress-activated signaling pathways and antioxidant response in CRC

Although oxidative reactions are often associated to the early phase of cancer, mainly in that they induce DNA damage, they may also be involved in the antioxidant response activation that can facilitate tumor progression and invasiveness, rather than having antitumor effects. Redox sensitive signaling pathways that are activated during inflammation in CRC are the principal actors of this phenomenon.

Besides its involvement in generating ROS during cancer initiation, NOX also plays an essential role in epithelial tumor cell proliferation and invasion. NOX1 is expressed in the human colon, depending on the degree of cell differentiation, its expression being up-regulated in adenomas and well-differentiated carcinomas. ${ }^{31}$ Conversely, ROS production by NOX1 can trigger the "angiogenic switch", by inducing angiogenic factors such as vascular endothelial growth factor and MMP-9, which promote epithelial tumor cell vascularization and proliferation. ${ }^{32}$

A large proportion of CRCs show constitutive activation of transcription factors, such as the redoxsensitive transcription factor $\mathrm{NF}-\kappa \mathrm{B}$, and the signal transduction molecules that are essential constituents of multiple inflammatory pathways. The aberrant activation of NF- $\mathrm{BB}$ is reported in over $50 \%$ of CRCs. ${ }^{33}$

Activation of NF- $\kappa \mathrm{B}$ via ROS generation during inflammation may support carcinogenesis. There is some evidence that antiapoptotic members of the B-cell leukemia/lymphoma 2 (Bcl2) family, which have an antioxidant function and are deregulated in cancer, are highly regulated NF- $\kappa \mathrm{B}$ target genes. ${ }^{34}$ Tumor cells with activated NF- $\mathrm{KB}$ acquire resistance to chemotherapics: inactivation of $\mathrm{NF}-\kappa \mathrm{B}$ greatly increases their sensitivity to these agents. ${ }^{35}$ The NF- $\kappa \mathrm{B}$ signaling pathway may be persistently activated in inflammatory cells and enterocytes, by Toll-like receptors (TLRs) and Nodlike receptors (NLRs), which are up-regulated in IBD. ${ }^{4}$ Activation of NF- $\kappa \mathrm{B}$ in myeloid cells (especially macrophages and dendritic cells of the intestinal lamina propria) determines the production of cytokines that act as growth factors for already altered enterocytes. ${ }^{33}$ 
In some cases, prolonged constitutive activity of $\mathrm{NF}-\kappa \mathrm{B}$ in the cancer cell nucleus is determined by the activity of STAT3, which has been found to be persistently activated in CRC. STAT3 belongs to a family of transcription factors that are involved in mediating cell growth and differentiation during immune and inflammatory processes. Certain STATs are specifically activated by Janus kinases (JAKs), a group of tyrosin kinase receptors whose ligands are cytokines. Genetic variations in JAK/STAT signaling pathway appear to influence CRC carcinogenesis. ${ }^{36}$ Interestingly, crosstalk between STAT3 and the mammalian target of rapamycin complex 1 (mTORC1), which controls anabolic cell growth and proliferation, can promote CRC in colitic mice. ${ }^{37}$

A study on Bacteroides enterotoxigenic fragilis, a commensal bacterium found in $20-35 \%$ of adults, found that this microbe promoted CRC formation in APC knockout mice by activating the Th17STAT3 dependent pathway mechanism; the use of anti-IL-17 antibodies, alone or combined with anti-IL-23 antibodies, significantly inhibited colon tumor formation. ${ }^{38}$

Very recent studies indicate that expression of redox-sensitive Nuclear Factor-Erythroid 2-Related Factor 2 (NRF2) is deregulated in various human cancers, including CRC, suggesting its possible involvement in carcinogenesis and therapy resistance. In normal cells, NRF2 plays a central role in cellular defense against oxidative stress. Upon its activation, NRF2 is set free from its own negative regulator, Kelch-like ECH-associated protein 1 (KEAP1), whereupon it translocates to the nucleus and binds to response elements on DNA, known as antioxidant response elements (AREs) or electrophile response elements (EpREs). It is able to do so by regulating the expression of genes encoding for redox balancing proteins, including heme oxygenase (HO)-1, SOD, enzymes necessary for GSH synthesis and recovery (glutamate-cysteine ligase catalytic/modulatory subunits GCLC/ GCLM, GSH-S-reductase), and thioredoxin (TXN) production (TXN-reductase-1, peroxiredoxin-1/4). NRF2 also regulates many other genes, particularly those involved in cell differentiation and proliferation. ${ }^{39}$

NRF2 is now considered to play a dual role in tumorigenesis: numerous reports indicate that activation of NRF2 can suppress carcinogenesis in many malignant tumors, especially at the earliest 
stages. ${ }^{39}$ Nevertheless, sustained activation of NRF2 occur in malignant tumors. In some cancers, mutations in $N r f 2$ or Keapl that enhance NRF2 activity have been associated with resistance to standard chemotherapy and poor survival. ${ }^{40}$ NRF2 may also promote cancer cell proliferation and tumorigenesis, by maintaining the redox balance and generating antioxidants in cancer cells. Recent studies have revealed that glutathione is critical for cell proliferation; therefore, enhancement of GSH synthesis is another important effect of NRF2 in accelerating cancer cell proliferation. ${ }^{41}$ Notably, NRF2 may participate in protection against inflammation by inhibiting $\mathrm{NF}-\kappa \mathrm{B} .{ }^{42}$ Conversely, it is also conceivable that NF- $\mathrm{KB}$ may activate NRF2 as a result of oxidative stress during inflammation. This latter effect has recently been observed in acute myeloid leukemia cells, in which NRF2 expression was reduced by NF- $\kappa$ B inhibitors. ${ }^{43}$

Among different NRF2 target proteins, HO-1 may have oncogenic potential in cancer cell proliferation, invasion, and resistance to anticancer treatment. HO-1 is a microsomal antioxidant enzyme, which is activated by a wide range of stimuli, including ROS, to catabolize free heme, thus preventing free-radical production through the Fenton reaction, resulting in decreased oxidative stress, attenuated inflammatory response, and inhibited apoptosis. ${ }^{39}$ HO-1 has been found to be upregulated in CRC compared to surrounding normal tissue; its overexpression in cancer cells provides cellular resistance against ROS-mediated anticancer therapies. ${ }^{44}$ Moreover, HO-1 accelerates tumor vascularization and increases cancer cells' metastatic potential because of its proangiogenic properties. ${ }^{45}$ Although HO-1 up-regulation has been found to exert pro-tumoral action, some studies report that CRC patients with high expression of this enzyme displayed longer overall survival times. HO-1 was found to decrease the migration and survival of cancer cells. ${ }^{46}$ Finally, its expression was reported to be associated with a lower rate of lymphatic invasion, and a tendency towards fewer lymph-node metastases; it was also found to be weaker at advanced stages of CRC, suggesting HO-1 may be a marker of genetic stability. ${ }^{47}$ Notably, the severity of DNA damage was found decreased at advanced stages of CRC, suggesting that oxidative reactions play different roles during different phases of carcinogenesis. ${ }^{48}$ 
Thus, the combination of enhanced NRF2 activity with HO-1 induction and antioxidant increase appears to be protective in normal cells, and beneficial during the premalignant phases of carcinogenesis. However, it is undesirable at later stages of CRC, when it increases both cell survival and the progression to fully malignant cancer cells, which finally become resistant to therapy.

\section{$\underline{\text { Conclusions and future directions }}$}

The substantial number of studies confirming the close relationship between chronic inflammation and oxidative stress in CRC carcinogenesis has encouraged many researchers to investigate the different pathways that may be involved in these processes, whose mutual cross-talk opens a new window for understanding these complicated phases of cancer development and progression. For many years, ROS have been thought to be important molecules, favoring early phases of CRC, while antioxidants have long been considered to be possible anticancer tools. However, numerous studies now show that an increased antioxidant status in cancer cells may activate survival and proliferation signaling pathways, making the cells resistant to the toxic effects of chemo/radiotherapy. This phenomenon is closely related to the activation of important molecules that modulate the cellular antioxidant response (Fig.1). Based on this observation, specific molecules involved in oxidative damage and inflammatory processes, which might enable the different phases of CRC to be characterized, still require investigation. Thus research targeting these molecules, and further investigating the antioxidant capacity of cancer cells, could be a promising approach for future therapeutic attempts in CRC treatment.

\section{$\underline{\text { Acknowledgements }}$}

The authors thank the CRT Foundation, Turin, and the University of Turin, Italy, for supporting this work.

Conflicts of interest: The authors state that there is not conflict of interest in the present paper. 


\section{$\underline{\text { References }}$}

1. Ferlay, J., I. Soerjomataram, R. Dikshit, et al. 2014. Cancer incidence and mortality worldwide: Sources, methods and major patterns in GLOBOCAN 2012. Int. J. Cancer. doi: 10.1002/ijc.29210. [Epub ahead of print].

2. Lovasz, B.D., L. Lakatos, A. Horvath, et al. 2013. Evolution of disease phenotype in adult and pediatric onset Crohn's disease in a population-based cohort. World J. Gastroenterol. 19: $2217-2226$

3. Kim, E.R. \& D.K. Chang. 2014. Colorectal cancer in inflammatory bowel disease: the risk, pathogenesis, prevention and diagnosis. World J. Gastroenterol. 20: 9872-9881.

4. Biasi, F., G. Leonarduzzi, P.I. Oteiza \& G. Poli. 2013. Inflammatory bowel disease: mechanisms, redox considerations, and therapeutic targets. Antioxid. Redox Signal. 19: $1711-1747$.

5. Perše, M. 2013. Oxidative stress in the pathogenesis of colorectal cancer: cause or consequence? Biomed. Res. Int. 2013:725710. doi: 10.1155/2013/725710.

6. Galon, J., A. Costes, F. Sanchez-Cabo, et al. 2006. Type, density, and location of immune cells within human colorectal tumors predict clinical outcome. Science. 313: 1960-1964.

7. Götlind, Y.Y., M. Fritsch Fredin, A.K. Kumawat, et al. 2013. Interplay between T(h)1 and $\mathrm{T}(\mathrm{h}) 17$ effector T-cell pathways in the pathogenesis of spontaneous colitis and colon cancer in the Gai2-deficient mouse. Int. Immunol. 25: 35-44.

8. Edin, S., M.L. Wikberg, A.M. Dahlin, et al. 2012. The distribution of macrophages with a $\mathrm{m} 1$ or $\mathrm{m} 2$ phenotype in relation to prognosis and the molecular characteristics of colorectal cancer. PLoS One. 7: e47045.

9. Galdiero, M.R., C. Garlanda, S. Jaillon, et al. 2013. Tumor associated macrophages and neutrophils in tumor progression. J. Cell Physiol. 228: 1404-1412. 
10. Shang, K., Y.P. Bai, C. Wang, et al. 2012. Crucial involvement of tumor-associated neutrophils in the regulation of chronic colitis-associated carcinogenesis in mice. PLoS One. 7: e51848.

11. Absenger, G., J. Szkandera, M. Pichler, et al. 2013. A derived neutrophil to lymphocyte ratio predicts clinical outcome in stage II and III colon cancer patients. Br. J. Cancer. 109: 395400.

12. Grivennikov, S.I. 2013. Inflammation and colorectal cancer: colitis-associated neoplasia. Semin. Immunopathol. 35: 229-244.

13. Wang, D. \& R.N. Dubois. 2010. The role of COX-2 in intestinal inflammation and colorectal cancer. Oncogene. 29: 781-788.

14. Bellamkonda, K., W. Sime \& A. Sjölander. 2014. The impact of inflammatory lipid mediators on colon cancer-initiating cells. Mol. Carcinog. doi: 10.1002/mc.22207. [Epub ahead of print].

15. De Simone, V., E. Franzè, G. Ronchetti, et al. 2014. Th17-type cytokines, IL-6 and TNF- $\alpha$ synergistically activate STAT3 and NF-kB to promote colorectal cancer cell growth. Oncogene. doi: 10.1038/onc.2014.286.

16. Klapproth, J.M. \& M. Sasaki. 2010. Bacterial induction of proinflammatory cytokines in inflammatory bowel disease. Inflamm. Bowel Dis. 16: 2173-2179.

17. Lee, Y.S., I. Choi, Y. Ning, et al. 2012. Interleukin-8 and its receptor CXCR2 in the tumour microenvironment promote colon cancer growth, progression and metastasis. Br. J. Cancer. 106: $1833-1841$.

18. Biasi, F., T. Guina, M. Maina, et al. 2012. Progressive increase of matrix metalloprotease-9 and interleukin-8 serum levels during carcinogenic process in human colorectal tract. PLoS One. 7: e41839.

19. Waldner, M.J., S. Foersch \& M.F. Neurath. 2012. Interleukin-6--a key regulator of colorectal cancer development. Int. J. Biol. Sci. 8: 1248-1253. 
20. Yu, L.Z., H.Y. Wang, S.P. Yang, et al. 2013. Expression of interleukin-22/STAT3 signaling pathway in ulcerative colitis and related carcinogenesis. World J. Gastroenterol. 19: 26382649.

21. Omrane, I., O. Baroudi, K. Bougatef, et al. 2014. Significant association between IL23R and IL17F polymorphisms and clinical features of colorectal cancer. Immunol. Lett. 158: 189194.

22. Fleming, N.I., R.N. Jorissen, D. Mouradov, et al. 2013. SMAD2, SMAD3 and SMAD4 mutations in colorectal cancer. Cancer Res. 73: 725-735.

23. Sipos, F. \& O. Galamb. 2012. Epithelial-to-mesenchymal and mesenchymal-to-epithelial transitions in the colon. World J. Gastroenterol. 18: 601-608.

24. Juhasz, A., Y. Ge, S. Markel, et al. 2009. Expression of NADPH oxidase homologues and accessory genes in human cancer cell lines, tumours and adjacent normal tissues. Free Rad. Res. 43: 523-532.

25. Kuwano, Y., K. Tominaga, T. Kawahara, et al. 2008. Tumor necrosis factor alpha activates transcription of the NADPH oxidase organizer 1 (NOXO1) gene and upregulates superoxide production in colon epithelial cells. Free Radic. Biol. Med. 45: 1642-1652.

26. Tardieu, D., J.P. Jaeg, A. Deloly, et al. 2000. The COX-2 inhibitor nimesulide suppresses superoxide and 8-hydroxy-deoxyguanosine formation, and stimulates apoptosis in mucosa during early colonic inflammation in rats. Carcinogenesis. 21: 973-976.

27. Dincer, Y., Y. Erzin, S. Himmetoglu, et al. 2007. Oxidative DNA damage and antioxidant activity in patients with inflammatory bowel disease. Dig. Dis. Sci. 52: 1636-1641.

28. Hussain, S.P., P. Amstad, K. Raja, et al. 2000. Increased p53 mutation load in noncancerous colon tissue from ulcerative colitis: a cancer-prone chronic inflammatory disease. Cancer Res. 60: 3333-3337. 
29. Nishikawa, M., N. Oshitani, T. Matsumoto, et al. 2005. Accumulation of mitochondrial DNA mutation with colorectal carcinogenesis in ulcerative colitis. Br. J. Cancer. 93: 331337.

30. Poli, G., R.J. Schaur, W.G. Siems \& G. Leonarduzzi. 2008. 4-hydroxynonenal: a membrane lipid oxidation product of medicinal interest. Med. Res. Rev. 28: 569-631.

31. Rokutan, K., T. Kawahara, Y. Kuwano, et al. 2006. NADPH oxidases in the gastrointestinal tract: a potential role of Nox1 in innate immune response and carcinogenesis. Antioxid. Redox Signal. 8: 1573-1582.

32. Arbiser, J.L., J. Petros, R. Klafter, et al. 2002. Reactive oxygen generated by Nox1 triggers the angiogenic switch. Proc. Natl. Acad. Sci. U S A. 99: 715-720.

33. Karin, M. 2006. Nuclear factor-kappaB in cancer development and progression. Nature. 441: 431-436.

34. Vogler, M. 2012. BCL2A1: the underdog in the BCL2 family. Cell Death Differ. 19: 67-74.

35. Berardi, R., E. Maccaroni, A. Mandolesi, et al. 2012. Nuclear factor- $\kappa$ B predicts outcome in locally advanced rectal cancer patients receiving neoadjuvant radio-chemotherapy. Dig. Liver Dis. 44: 617-622.

36. Slattery, M.L., A. Lundgreen, S.A. Kadlubar, et al. 2013. JAK/STAT/SOCS-signaling pathway and colon and rectal cancer. Mol. Carcinog. 52: 155-166.

37. He, Z., X. He, Z. Chen, et al. 2014. Activation of the mTORC1 and STAT3 pathways promotes the malignant transformation of colitis in mice. Oncol. Rep. doi: 10.3892/or.2014.3421. [Epub ahead of print].

38. Wu, S., K.J. Rhee, E. Albesiano, et al. 2009. A human colonic commensal promotes colon tumorigenesis via activation of T helper type $17 \mathrm{~T}$ cell responses. Nat. Med. 15: 1016-1022.

39. Geismann, C., A. Arlt, S. Sebens \& H. Schäfer. 2014. Cytoprotection "gone astray": Nrf2 and its role in cancer. Onco Targets Ther. 7: 1497-1518. 
40. Gañán-Gómez, I., Y. Wei, H. Yang, et al. 2013. Oncogenic functions of the transcription factor Nrf2. Free Radic. Biol. Med. 65: 750-764.

41. Gorrini, C., I.S. Harris \& T.W. Mak. 2013. Modulation of oxidative stress as an anticancer strategy. Nat. Rev. Drug Discov. 12: 931-947.

42. Kim, J., Y.N. Cha \& Y.J. Surh. 2010. A protective role of nuclear factor-erythroid 2-related factor-2 (Nrf2) in inflammatory disorders. Mutat. Res. 690: 12-23.

43. Rushworth, S.A., L. Zaitseva, M.Y. Murray, et al. 2012. The high Nrf2 expression in human acute myeloid leukemia is driven by NF- $\mathrm{BB}$ and underlies its chemo-resistance. Blood. 120: 5188-5198.

44. Yin, H., J. Fang, L. Liao, et al. 2014. Upregulation of heme oxygenase-1 in colorectal cancer patients with increased circulation carbon monoxide levels, potentially affects chemotherapeutic sensitivity. BMC Cancer. 14: 436.

45. Was, H., J. Dulak \& A. Jozkowicz. 2010. Heme oxygenase-1 in tumor biology and therapy. Curr. Drug Targets. 11: 1551-1570.

46. Andrés, N.C., M.E. Fermento, N.A. Gandini, et al. 2014. Heme oxygenase-1 has antitumoral effects in colorectal cancer: Involvement of p53. Exp. Mol. Pathol. 97: 321-331.

47. Becker, J.C., H. Fukui, Y. Imai, et al. 2007. Colonic expression of heme oxygenase-1 is associated with a better long-term survival in patients with colorectal cancer. Scand. J. Gastroenterol. 42: 852-858.

48. Ribeiro, M.L., D.G. Priolli, D.D. Miranda, et al. 2008. Analysis of oxidative DNA damage in patients with colorectal cancer. Clin. Colorectal Cancer. 7: 267-272. 


\section{Figure Legend}

Figure 1. Inflammation and oxidative reactions are implicated in different phases of colorectal carcinogenesis.

Pre-existing inflammation can result in mutations and epigenetic changes that favor tumor initiation. Reactive Oxygen/Nitrogen species (ROS/RNS), pro-inflammatory cytokines, growth factors and prostaglandins produced by activated inflammatory/immune cells, induce mutations and genomic alterations resulting in the appearance of premalignant cells (see "damaged enterocyte") that proliferate and survive in response to these stimuli. In such microenvironment, during early stages of malignant transformation, inflammatory infiltrate is typically consisted of cells that express pro-inflammatory phenotype, such as Tumor Associated Macrophages (TAM) 1, Tumor Associated Neutrophils (TAN) 1 and T helper (Th) 17 lymphocytes. These cells are responsible for transcription factors Nuclear Factor- $\mathrm{B}(\mathrm{NF}-\kappa \mathrm{B})$ and Signal Transducer and Activator of Transcription (STAT) 3 activation, and also for NADPH oxidase (NOX) up-regulation, which represent the major key events implicated in the pathogenesis of inflammation-associated tumor development. Furthermore, activation of Toll-like receptors (TLRs) and Nod-like receptors (NLRs) by intestinal microbiota may contribute to inflammation through NF- $\kappa$ B and STAT3 activation. NOX and mitochondria are chief molecules responsible for ROS generation and related oxidation products able to induce structural and functional damage of important molecules involved in cell growth and proliferation.

Normally, oxidative insult in enterocytes is counteracted by cellular antioxidant defenses, mainly through the up-regulation the redox sensitive Nuclear Factor-Erythroid 2-Related Factor 2 (NRF2), which induces antioxidant molecules, such as glutathione (GSH), heme oxygenase (HO)-1, superoxide dismutase (SOD) and thioredoxin (TXN).

Conversely, when cells acquire malignant phenotype (see "tumor cell"), the increased antioxidant response and the elevated production of antioxidants enables them to progress and facilitate invasion. Notably, at these cancer stages inflammatory infiltrate is replaced by cells with pro- 
tumoral activity, i.e. TAM 2, TAN 2 and Th1, which mainly synthesized different growth factors and proteinases sustaining tumor survival and invasiveness.

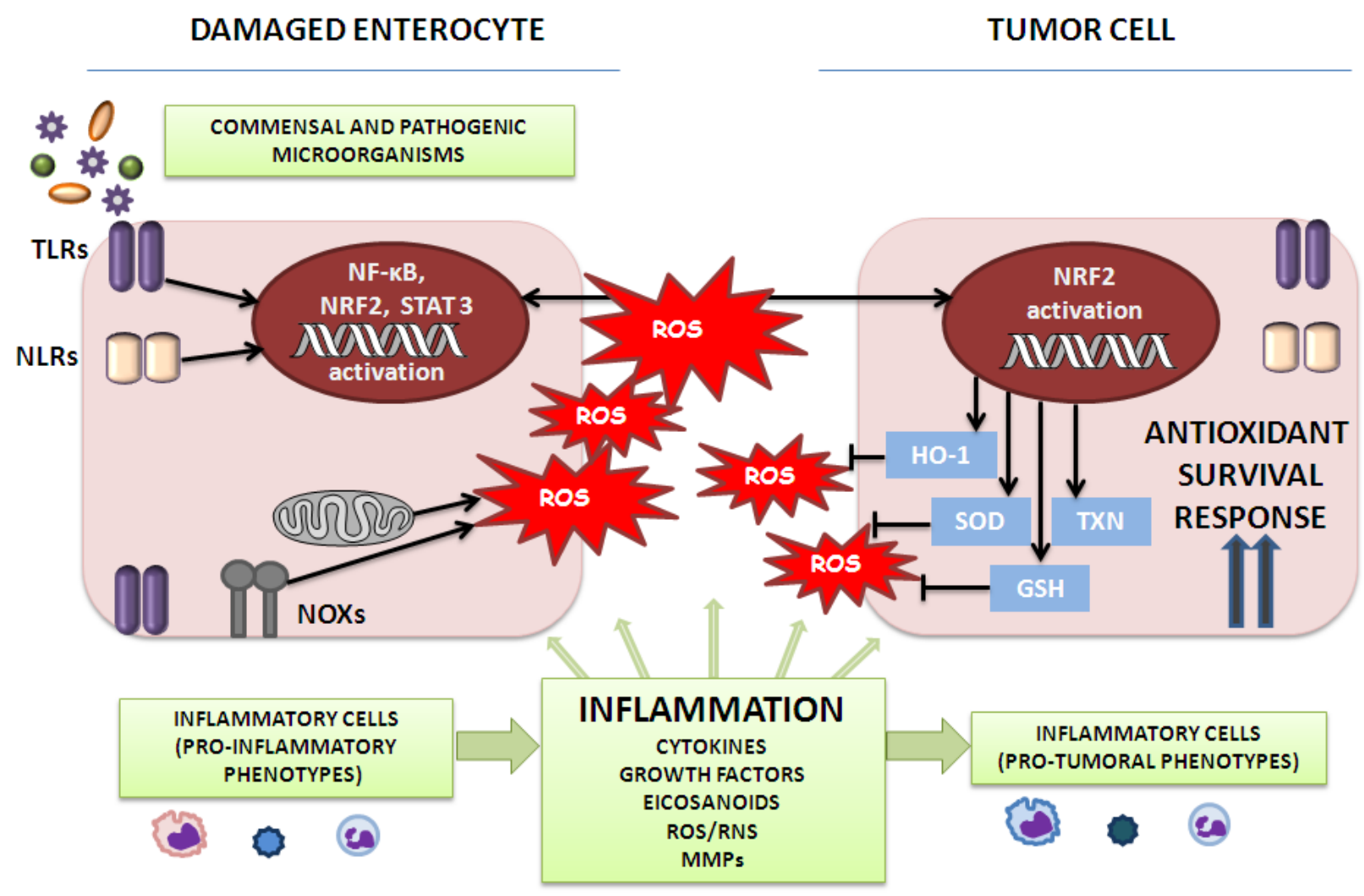

TAM1 $\quad$ Th17

TAM2 (6) Th1 TAN 